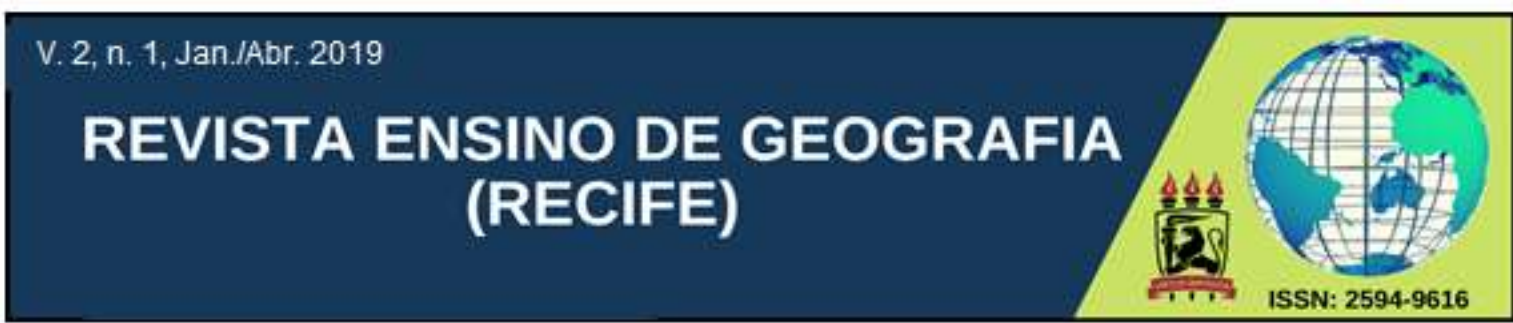

\title{
PLUVIÔMETRO COM GARRAFAS PET'S: ANÁLISE DAS PRÁTICAS METODOLÓGICAS USADAS COM MATERIAIS ALTERNATIVOS COMO FACILITADOR DO ENSINO-APRENDIZAGEM DE CLIMATOLOGIA
}

\author{
Rodrigo Nascimento Bentes \\ Pós-graduado em gestão e docência no ensino superior pela (FAM) \\ rodrigogeouepa20@gmail.com \\ ORCID iD: https://orcid.org/0000-0002-1640-0899
}

Artigo recebido em 23/04/2019 e aceito em 28/06/2019

\begin{abstract}
RESUMO: O presente trabalho tem como objetivo analisar os recursos metodológicos utilizados pelo docente em sala de aula mediante o uso de materiais alternativos para o ensino da disciplina Climatologia, com a construção de um pluviômetro feito com garrafas Pet's, onde, como propostas metodológicas possibilitou compreender os princípios sobre pluviosidade e capacidade pluviométrica no município de Barcarena, no Estado do Pará. A partir desta atividade em sala de aula, foi possível facilitar o conteúdo disciplinar de forma a não se comparar com o padrão tradicional de ensino e dinamizando as atividades de forma contextualizada, o que enriquece metodologicamente o conteúdo quando ministrado pelo professor. Com a construção deste equipamento alternativo, alcançaram-se dados importantes para compreender sobre a pluviosidade nesta região, sendo o município pertencente à região amazônica, onde ocorrem fatores climáticos e meteorológicos diferenciados de outras regiões do país. Com essa forma de ensinar, o processo de ensino-aprendizagem é garantido e torna-se eficaz para o desenvolvimento critico do discente, sobre a determinada temática abordada, neste caso, o estudo da pluviosidade em Climatologia.
\end{abstract}

Palavras-chave: materiais alternativos, climatologia, ensino.

\section{PLUVIOMETER WITH PET'S BOTTLES: ANALYSIS OF THE METHODOLOGICAL PRACTICES USED WITH ALTERNATIVE MATERIALS AS FACILITATOR OF THE TEACHING-LEARNING OF CLIMATOLOGY}

\begin{abstract}
The present work aims to analyze the methodological resources used by the classroom teacher through the use of alternative materials for teaching the discipline Climatology, with the construction of a rain gauge made with Pet's bottles, where as methodological proposals made possible to understand the principles on rainfall and rainfall capacity in the municipality of Barcarena, in the State of Pará. From this activity in the classroom, it was possible to facilitate the disciplinary content in a way that does not compare with the traditional pattern of teaching and dynamising the activities in a contextualized way, which methodically enriches the content when taught by the teacher. With the construction of this alternative equipment, important data were obtained to understand the rainfall in this region, being the municipality belonging to the Amazon region, where climatic and meteorological factors differ from other regions of the country. With this way of teaching, the teaching-learning process is guaranteed and becomes effective for the critical development of the student, on the specific subject addressed, in this case, the study of rainfall in Climatology.
\end{abstract}

Keywords: alternative materials, climatology, teaching. 


\section{INTRODUÇÃO}

As grandes e constantes inovações que ocorrem na natureza e os papeis contributivos do homem para a transformação do seu meio, se dão pelas intenções nas quais são atribuídas na necessidade do ser humano em entender sobre os recursos que a natureza dispõe e o que pode ser geradas para seu retorno. Nestas condições entre a relação homem-natureza, o estudo da geografia trás para a formação crítica pessoal e coletiva da sociedade, atribuições que, esta disciplina pode contribuir socialmente, entre a motivação e o sentido de cada homem atribuir de funções para meio à qual vivem.

Estas inovações trazem ao ensino da disciplina geografia grandes desafios que para o docente, deve enfrentar como oportunidades de melhor dinamizar determinado conceito ou conteúdo, aplicando nas diversas temáticas expostas, o cumprimento curricular proposta por tal área de competência contextualizando-a.

A climatologia, por exemplo, que no nível superior é vista de forma mais rebuscada perante as distribuições e linhas teóricas enquanto climatologia clássica e climatologia dinâmica, que estudam os diversos fatores que contribuem para a sua concepção e definição, que segundo Grimm (2017) é tida como o estudo dos fenômenos atmosféricos do ponto de vista de suas propriedades estatísticas (médias e variabilidade) caracterizando o clima em função da localização geográfica, estação do ano, hora do dia, como temáticas de estudo, inserindo-a enquanto disciplina complementar a geografia física.

Para melhor contribuir e facilitar o ensino aprendizagem, o mediador pode recorrer à utilização de recursos pedagógicos para enriquecer a abordagem teórica de determinado conteúdo, neste caso, melhor favorecer o ensino da temática da climatologia em sala de aula. Pimenta (2002) enfatiza que a prática do professor é essencial para promover o ensinoaprendizagem junto ao aluno, ou seja, através do ensino, há a garantia da aprendizagem como consequência a ação de ensinar.

Por esta ação complementar que o processo de ensino aprendizagem promove maior oportunidade de se conhecer novas realidades, em vista do desenvolvimento da concepção cientifica, condicionando em uma mudança de realidade e intervenção sobre este ponto, no campo do conhecimento (MELLO, 2012).

O presente trabalho teve como objetivo principal analisar os recursos metodológicos através de relato de experiência obtidos das atividades propostas pelo docente, através do uso 
de materiais alternativos para o ensino da disciplina Climatologia com alunos de licenciatura em geografia da Universidade do Estado do Pará, a partir do estudo sobre pluviosidade, com a construção de um pluviômetro de baixo custo para facilitar a explanação sobre tal conteúdo disciplinar de forma dinamizada.

\section{REVISÃO BIBLIOGRÁFICA}

O ensino de climatologia que, acompanhada da meteorologia, são conteúdos já apresentados aos alunos na geografia, nas series iniciais que, torna-se de difícil compreensão à medida que vem trazendo novas informações conceituais e definições junto às relações entre clima e tempo em dinâmica natural e também através da ação antrópica no meio.

Para melhor facilitar esta temática, Marquardt \& Sousa (2015), propõe que estas conceituações fluem melhor a partir das aulas práticas nas estações meteorológicas despertando o interesse dos alunos em um espaço diferenciado. Entretanto, com a falta áreas de pesquisa e equipamentos mais eficazes e com aparatos tecnológicos, podem-se aplicar estas mesmas definições e relações conceituais através do uso de materiais de fácil acesso, baixo custo e disponível no dia-a-dia de cada aluno, no seu contexto.

O uso de recursos alternativos ou de baixo custo são umas das diversas ações metodológicas que podem ser aplicadas no estudo de climatologia geográfica, onde, mediante a aplicação destes métodos, aumenta a criticidade e intelectualidade dos alunos, fazendo-os questionar sobre sua participação enquanto ser vivente no espaço onde vivem.

A precipitação pluviométrica, característica presente na variação do espaço geográfico, e elemento meteorológico dos fatores climáticos existentes, são expressos nas abordagens climatológicas que por sua vez, atribuem diversas conceptualizações e aplicações de referenciais próprios da área tendo uma linguagem técnica e própria que em alguns casos, dificultam a assimilação pela extensão de conteúdo e não compreendidas por parte dos alunos quando não entendido suas definições.

Para mensurar o nível de precipitação em determinada localidade, usa-se o equipamento denominado de pluviômetro que, por conseguinte, inferido a cálculos matemáticos dados para compor uma medida aritmética.

O pluviômetro tem como objetivo segundo Cacilhas et al (2018) "recolher e medir, em milímetros lineares, a quantidade de líquidos ou sólidos (chuva, neve, granizo) precipitados 
durante um determinado tempo e local. Medido em milímetros, é a somatória da precipitação num determinado local durante um período de tempo estabelecido".

A necessidade de determinados conteúdos serem mais bem compreendidos pelos alunos, mostra a importância que recursos metodológicos usados como ferramentas complementares contribuem para o ministrante ao perpassar, neste caso o ensino de climatologia em perspectiva geográfica, ressaltando a importância de uma estação meteorológica e climática para fazer estudos detalhados sobre o nível pluviométrico de cada região e outras aplicações no contexto próximo aos dos alunos, no Estado, país e, por conseguinte, uma análise global. Marandola Jr. (2009) afirma que o homem, é peça fundamental para o entendimento do tempo e clima, tornando-se alvo do próprio estudo climatológico, e também responsável pelos processos ocorridos pela atmosfera, nos diversos níveis de distribuições climáticas dadas os diversos pontos do globo terrestre.

O professor como mediador dessa didática de ensino de forma contextualizada vê-se no desafio de construir ferramentas, de baixo custo ou não, para aumentar as formas metodológicas de ministrar e contribuir para a formação satisfatória dos alunos, abordando de maneira lúdica e divertida, o ensino de forma mais facilitada, instigando o aluno a participação e complementação de tal assunto abordado. Segundo Lira et al (2015, p.5), expõem que:

\footnotetext{
A experiência pedagógica do professor além de dominar a linguagem, conceitos, procedimentos teóricos e a transposição da pesquisa acadêmica na prática escolar ele tem um encontro pedagógico com os alunos e automaticamente o mesmo, age de forma comunicativa com os alunos através do conteúdo. Sendo o professor um elemento que veicula o conteúdo através das metodologias de ensino.
}

Sousa et al (2015) contribuem explicando que a didática e as metodologias de ensino e aprendizagem formam uma unidade, mantendo entre si relações recíprocas, cabendo ao professor ter suas metodologias próprias de trabalho, viabilizando momentos propícios para a aquisição de conhecimento aos alunos.

Através desses pressupostos, é conveniente o uso de materiais alternativo para o ensino de climatologia, no ensino de pluviosidade, analisando as formas de interpretação sobre tal assunto, mesmo no ambiente acadêmico, facilitando-o. 


\section{BREVE CONTEXTUALIZAÇÃO DO TERRITORIO DE BARCARENA-PARÁ QUANTO ANÁLISE PLUVIOMETRICA}

O município de análise, Barcarena, está localizado na mesorregião metropolitana de Belém, mais precisamente na microrregião de Belém, Localizando-se a uma latitude $01^{\circ} 30^{\prime} 21^{\prime \prime}$ sul e a uma longitude48 37 '33" oeste (Mapa 1); estando a uma altitude de 15 metros em relação do nível do mar. Segundo o IBGE, sua população está estimada em 109.975 habitantes (estimativa do ano 2013). (PREFEITURA MUNICIPIAL DE BARCARENA, 2019)

Mapa 1- Mapa temático do município de Barcarena-Pará

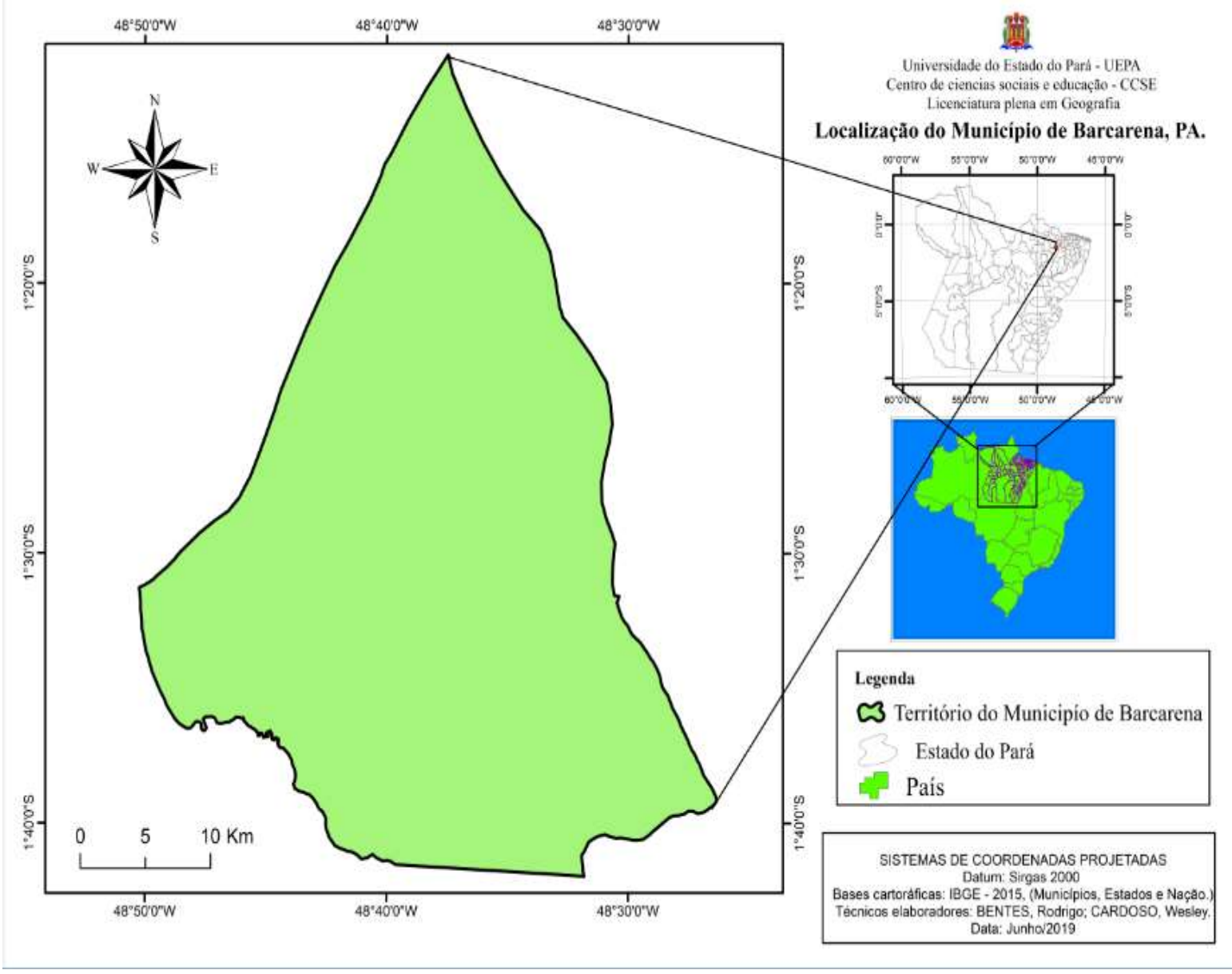

Fonte: Bentes \& Cardoso (2019)

Sobre os aspectos pluviométricos, Barcarena pertencente à região tropical, apresenta características quanto à temperatura, umidade relativa e ventos, apresentam particularidades, onde segundo Fisch et al (1998), apresenta precipitação média de aproximadamente de 2.300 mm ao ano. A Amazônia não apresenta homogeneidade no que se refere o regime de 
precipitação, variando espacialmente e agindo de forma interativa aos diferentes sistemas presentes como citado por Rocha (1991), em zona de Convergência Intertropical sobre o Oceano Atlântico Equatorial.

Segundo Albuquerque et al (2010), A maior parte dos Estados da Amazônia apresentam o período chuvoso durante os meses de outubro a maio, e a média de acumulação variam entre $600 \mathrm{~mm}$ a $2100 \mathrm{~mm}$. Já o período seco, é presente em grande parte da Amazônia ocorre durante os meses de junho, julho e agosto.

Este período caracteriza-se pela baixa pluviosidade (chuvas abaixo de $50 \mathrm{~mm} / \mathrm{mês}$ ) e longos períodos de estiagem, nas quais atingem principalmente os Estados do Acre, Rondônia, Mato Grosso, Tocantins, centro sul do Maranhão, e sul do Amazonas e do Pará (ALBUQUERQUE ET AL, 2010). A chuva neste período ocorre em forma de pancadas isoladas e em áreas esparsas (SOUZA; AMBRIZZI, 2003).

\section{METODOLOGIA}

A construção do pluviômetro com materiais alternativos foi realizada mediante ao módulo de climatologia, na disciplina de geografia, na universidade do Estado do Pará, campus de Barcarena, para os alunos do $2^{\circ}$ semestre do curso de geografia.

Inicialmente, desenvolveu-se o conteúdo temático para adquirir as primeiras noções sobre o tema de climatologia e favorecer o desenvolvimento crítica sobre o determinado assunto. Após o mediador abordar o conteúdo sobre a dinamicidade da climatologia e desenvolver diferenças sobre tempo e clima, abordando-os e distinguindo-os citou a pluviosidade como uma das características presentes nos estudos climatológicos e propôs a construção de pluviômetros com matérias alternativos como garrafas pet para analisar o índice pluviométrico da região.

Posteriormente, aplicou de maneira individual a construção de um pluviômetro, com os seguintes materiais: uma garrafa pet, fita adesiva, estilete ou tesoura, seixo e uma régua, orientando a construção do equipamento e explicando a forma de uso para adquirir no fim das atividades dados mensuráveis para análise e pontos de referência para o tema ministrado pelo mesmo. (Imagem 1) 
Imagem 1 - pluviômetro construído com materiais alternativos.

Fonte: imagem captada pelo autor.

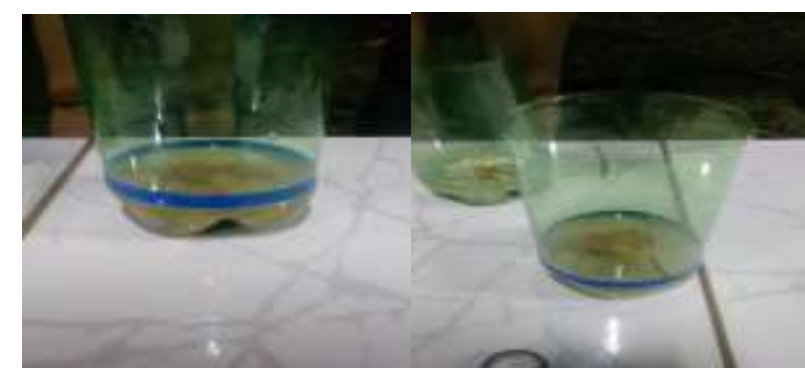

Foi sugerida pelo docente a construção de uma tabela para anotação e elaboração no fim da atividade que concluiria a avaliação com os seguintes pontos: data de exposição do pluviômetro na área escolhida, o horário inicial de exposição, o horário do término da exposição, os milímetros obtidos pela captação de agua da chuva e a observação sobre o tempo no dia de coleta de dados. (Quadro 1)

\begin{tabular}{|c|c|c|c|c|}
\hline \multicolumn{5}{|c|}{ Quadro 1: Modelo demonstrativo com a coleta de dados obtidos do pluviômetro; } \\
\hline Data & Início (horas) & Fim (horas) & Milímetros & $\begin{array}{c}\text { Obs.: (como está o tempo no } \\
\text { momento) }\end{array}$ \\
\hline xxxxxxxxxx & xxxxxxxxxxx & xxxxxxxxxxxx & xxxxxxxxxxxx & xxxxxxxxxxxx \\
\hline
\end{tabular}

Fonte: organizado pelo autor

\section{RESULTADOS E DISCUSSÃO}

Os dados obtidos através das coletas ocorreram em 11 dias. Do dia 26/09/2017 a 06/10/2017, intervalo a qual se dava o curso modular da disciplina de climatologia para a turma de geografia em questão. Os dados foram desenvolvidos a partir da construção do pluviômetro no dia 26/09/2017, onde apresenta informação de coleta as 23:00 até 13:30. A partir das datas posteriores analisadas sempre as 07:15 da manhã e finalizada sempre as 07:00, com 15 minutos de intervalo de início de cada coleta. 
A tabela a seguir exibe a coleta realizada nos dias definidos (Quadro 2):

\begin{tabular}{|c|c|c|c|c|}
\hline \multicolumn{5}{|c|}{ Quadro 2: coleta de dados obtidos do pluviômetro } \\
\hline Data & Início (horas) & Fim (horas) & Milímetros & $\begin{array}{c}\text { Obs.: (como está o tempo no } \\
\text { momento) }\end{array}$ \\
\hline $\mathbf{2 6 / 0 9 / 2 0 1 7}$ & $23: 00$ & $13: 30$ & 0 & CÉU NUBLADO \\
\hline $\mathbf{2 7 / 0 9 / 2 0 1 7}$ & $07: 15$ & $07: 00$ & 4 & CÉU ABERTO \\
\hline $\mathbf{2 8 / 0 9 / 2 0 1 7}$ & $07: 15$ & $07: 00$ & 3 & CÉU ABERTO \\
\hline $\mathbf{2 9 / 0 9 / 2 0 1 7}$ & $07: 15$ & $07: 00$ & 0 & CÉU ABERTO \\
\hline $\mathbf{3 0 / 0 9 / 2 0 1 7}$ & $07: 15$ & $07: 00$ & 0 & CÉU ABERTO \\
\hline $\mathbf{0 1 / 1 0 / 2 0 1 7}$ & $07: 15$ & $07: 00$ & 0 & CÉU ABERTO \\
\hline $\mathbf{0 2 / 1 0 / 2 0 1 7}$ & $07: 15$ & $07: 00$ & 0 & CÉU ABERTO \\
\hline $\mathbf{0 3 / 1 0 / 2 0 1 7}$ & $07: 15$ & $07: 00$ & 0 & CÉU NUBLADO \\
\hline $\mathbf{0 4 / 1 0 / 2 0 1 7}$ & $07: 15$ & $07: 00$ & 3 & CÉU NUBLADO \\
\hline $\mathbf{0 5 / 1 0 / 2 0 1 7}$ & $07: 15$ & $07: 00$ & 4 & CÉU NUBLADO \\
\hline $\mathbf{0 6 / 1 0 / 2 0 1 7}$ & $07: 15$ & $07: 00$ & 0 & \\
\hline & & & & 0 \\
\hline
\end{tabular}

Fonte: organizado pelo autor

O Referido mês de coleta de dados pluviométricos com o equipamento alternativo foi no período de setembro/outubro, sendo estas, meses de transição do período de baixos índices pluviométricos para o de alto índice, sendo outubro, o mês que inicia o mês de formações chuvosas, por este motivo, as manifestações de tempo aberto em grande parte dos dias coletados e tempo nublado em outros, com coleta variada, dos índices de chuva, quando analisadas do dia 26/09/2017 a 06/10/2017.

Os dias de conferência dos milímetros coletados no pluviômetro, variaram de $0 \mathrm{~mm}$ a $4 \mathrm{~mm}$ durante os dias referidos, e durante 24 horas de prazo se repetia continuamente a coleta, neste momento se observavam o tempo no momento, e os horários previstos. Somente no primeiro dia de coleta foi realizada uma coleta inicial diferente dos dias restantes, pois, foi posicionado horas depois da confecção em sala de aula.

Sobre o enfoque educacional, foi coletada a opinião de alguns alunos no que diz respeito o uso deste recurso metodológico para melhor complementar o ensino de Climatologia, no assunto sobre os processos de pluviosidade. 
Destacam-se as seguintes respostas, após o termino da disciplina em questão:

\begin{abstract}
"Nossa! foi muito interessante, gostei de poder construir algo que mesmo simples, possibilitou meu entendimento sobre o assunto! pensei que iria me enrolar todo, mas, no fim deu tudo certo! professor fez super certo em fazer algo simples, para ensinar esta disciplina que parecer ser bem difícil em alguns momentos" (B.D).
\end{abstract}

Sobre o pluviômetro construído, o aluno fez comentários positivos quanto os materiais de uso, e sobre a disciplina:

\begin{abstract}
"Quando o professor sugeriu a construção de um pluviômetro, logo pensei em algo mirabolante e surreal, bem tecnológico, mais quando (termo regional utilizado em nossa localidade), trouxemos garrafas pets e seixo para construir! bem simples e de uma grande importância. A disciplina foi muito agradável, e os termos conceituais após essa dinâmica, ficaram mais simplificados no meu entendimento" (M.F. O).
\end{abstract}

Outro entrevistado pontuou que deseja quando estiver ministrando, utilizar estes recursos metodológicos para os alunos das series iniciais quando tiver contato com as primeiras informações da disciplina climatologia. $\mathrm{O}$ aluno afirmou:

\footnotetext{
"O uso destes recursos foi importante para entender mais sobre as chuvas, seus períodos e características no nosso município. Percebi que os conceitos envolvendo pluviosidade e os estudos de climatologia são importantes para entender a influencia do clima e dos fatores formativos da terra, para os acontecimentos de diversos fenômenos naturais. quando estiver formado e ministrando irei com certeza utilizar este recurso, o pluviômetro alternativo, para melhor aproximar a disciplina ao meu aluno" (R.B).
}

Tendo em vista os relatos coletados, somente se enfatiza como positivo o uso do pluviômetro alternativo para o processo de desenvolvimento de ensino-aprendizagem, também, no ambiente formativo da Universidade para perpassar para as series anteriores de formação. O professor é capaz de tornar mais atraente uma determinada disciplina, quando consegue utilizar recursos que possa aproximar a realidade do aluno, em determinada situação do cotidiano destes e percebendo as dificuldades enfrentadas durante o ensinar em sala de aula.

\title{
5 CONSIDERAÇÕES FINAIS
}

O uso de materiais alternativos como recursos pedagógicos na metodologia do docente amplia a possibilidade em disponibilizar conteúdos complexos de forma simplificada, tendo em vista a construção da criticidade do aluno em meio às ações pedagógicas utilizadas. 
Quanto ao uso do pluviômetro, o objetivo de compreender a precipitação como uma condição que está inserida ao clima, como condicionante de fatores climáticos apresenta relevância e importância de ser bem mais conhecido e compreendido, não somente por climatologistas e meteorologistas, mas sim por qualquer pessoa que se interesse por este assunto e deseja entender o efeito da pluviosidade na sua região ou até mesmo, em escala global.

As noções sobre pluviosidade são importantes, pois com a baixa ou alta pluviosidade processos como, irrigação, agricultura, abastecimento de água nas residências e diversos processos a qual o homem depende necessariamente da presença de chuvas e recursos hídricos abundantes são necessários, sendo fundamentais para o aprendizado e compreensão do ciclo, no caso, noções sobre pluviosidade.

A instrumentação, necessária para análise do nível, em milímetros, do total precipitado em determinado local a determinado tempo, possibilita junto à ação climatológica, fazer previas sobre o tempo e mensurar por sua vez quantidade precipitada, tendo conhecimento do quanto e onde há uma capacidade de precipitação mais abundante, entre outras funções necessárias e enriquecedoras para a meteorologia e climatologia.

Como auxiliar enquanto recurso metodológico, o uso de materiais alternativos é necessário como facilitador e que complementa, enquanto ensino em geografia e climatologia, uma ótima ferramenta que possibilita uma definição mais segura, como no caso definição sobre pluviosidade, enriquecedora no processo de ensino aprendizagem e que contribua para a formação crítica do aluno universitário, neste caso, perpassando estas práticas metodológicas futuramente para os alunos, quando formados e de fato, docentes em geografia.

\section{REFERENCIAS}

ALBUQUERQUE, Monik Fernandes de. SOUZA, Everaldo Barreiros de. OLIVEIRA, Maria do Carmo Felipe de. Júnior, José Augusto de Souza. Precipitação nas mesorregiões do Estado do Pará: climatologia, variabilidade e tendências nas últimas décadas (1978-2008). Revista Brasileira de Climatologia. Ano 6 - Vol. 6 - Junho/ 2010.

CACILHAS, Leonardo Edemir; HAMES, Paulo Ricardo; GONÇALVES, Francilaine. Pluviômetro de Roda d'água. Florianópolis SC, [entre 2005 a 2015]. Acesso em: 15 Mar.2018

FISCH, G.; MARENGO, J. M.; NOBRE, C. A. Uma revisão geral sobre o clima da Amazônia. Acta Amazônica, v.28, n.2, p.101-126. 1998. 
LIRA, Francisco Sousa. MACHADO, Maria José Jorge. SANTOS, Swhelen Saraiva. SOUSA, Romário Rosa de. O Ensino de Climatologia Geográfica a partir da utilização de Uma Estação Meteorológica de Baixo Custo. VII encontro Nacional de Ensino de Geografia, out. Catalão (GO). 2015.

GRIMM, Alice Marlene. Apostila de meteorologia. Disponível em $<$ HTTP://fisica.ufpr.br/grimm/aposmeteo/>. Elaborado pela professora Dra. Alice Marlene Grimm da UFPR. Acesso em: 27.set.2017. Apostila.

MARANDOLA JR, E. Tangenciando a vulnerabilidade. In: HOGAN, D. J. \& MARANDOLA JR, E. (org.) Populações e mudanças climáticas: dimensões humanas das mudanças ambientais globais, CAMPINAS: NEPO/Unicamp; Brasília: UNFPA. P.: 29-52. 2009. Disponível em <http://:/Users/windows7/Downloads/Cap_11_PrecPluv.pdf.> Acesso em: 28. Set.2017.

MELLO, Márcia Cristina de Oliveira. Da teoria à prática do ensino da Geografia. Conteúdos e didáticas de geografia. UNESP, 2012.

ROCHA, E.J.P. Balanço de umidade na Amazônia durante o experimento FLUAMAZON. 1991. Dissertação (MSc. em Meteorologia) - Departamento de Ciências Atmosféricas-IAG-USP, São Paulo, 1991.

SOUZA, E. B.; AMBRIZZI, T. Pentad precipitation climatology over Brazil and the associated atmospheric mechanisms. Climanálise, São José dos Campos, v. 5, n.6 p. 36-44. jan. 2003.

PIMENTA, Selma Garrido. O estágio na formação de professores: unidade teoria e prática? 5. ed. São Paulo: Cortez, 2002.

PREFEITURA MUNICIPAL DE BARCARENA. Geografia do Município de Barcarena. Disponível em: <http://www.barcarena.pa.gov.br/portal/pagina?id=10\&url=geografia $>$. Acesso em: 27.Jun.2019.

MARQUARDT, Djane Gomes; SOUSA, Romário Rosa de. Oficina de climatologia uma forma diferente de ensino e aprendizagem com acadêmicos do curso de segunda licenciatura em geografia - PARFOR - CUA/UFMT. Variabilidade e susceptibilidade climática: Implicações Ecossistêmicas e Sociais. Goiânia (GO)/UFG. Out. 2015

SOUSA, Romário Rosa De. RODRIGUES, Luciene Inácio. PAULO, Celso Aparecido De. Estação meteorológica de baixo custo. Variabilidade e susceptibilidade climática: Implicações Ecossistêmicas e Sociais. Goiânia (GO)/UFG. Out.2015. 